\title{
Optimization of Spirulina Biofilm for in-situ Heavy Metals Detection with Microfluidic-Acoustic Sensor and AFM
}

\author{
Tekaya N. ${ }^{1,2,3,5}$, Tarbague $H_{.}{ }^{1}$, Moroté $F^{2}{ }^{2}$, Gammoudi I. ${ }^{1}$, Sakly $N^{3}{ }^{3}$, Ben Ouada Hat. ${ }^{4}$, \\ Raimbault V. ${ }^{1}$, Rebière $D .{ }^{1}$, Ouada Haf. Ben ${ }^{3}$, Jaffrezic-Renault $N .{ }^{5}$, Lagarde $F^{5}{ }^{5}$, Dejous C. ${ }^{1}$, \\ Cohen-Bouhacina $T^{2}$ \\ ${ }^{1}$ Univ. Bordeaux1, Laboratoire IMS, CNRS UMR 5218, 351 crs Libération, 33405 Talence, France \\ ${ }^{2}$ Univ. Bordeaux1, LOMA, CNRS UMR 5798, 351 crs Libération, 33405 Talence, France \\ ${ }^{3}$ Univ. Monastir, LIMA, Faculté des Sciences de Monastir, Bd Environnement, 5000 Monastir, Tunisia \\ ${ }^{4}$ Univ. Monastir, INSTM, rte Khniss, 5000 Monastir, Tunisia \\ ${ }^{5}$ Univ. Lyon, LSA, CNRS/ENS/UCBL UMR 5280, 43 Bd 11 Nov 1918, 69622 Villeurbanne, France \\ tekayanadeje@yahoo.fr
}

\begin{abstract}
:
Arthrospira platensis, called spirulina (Sp), is known to bind a wide range of heavy metals [1]. We propose an innovative approach, based on Spirulina as bioreceptor combined with highly sensitive Love wave platform for fast detection of heavy metals in solution.

Our goal is to optimize the biofunctionalization of the sensor surface with microalgae, based on realtime responses of the acoustic sensor during Spirulina immobilization then heavy metals detection, combined with atomic force microscopy characterization to improve understanding of interaction phenomena. Both methods have proved the efficiency of a microfluidic chip to control the hydrodynamical flow, resulting in a biofunctional layer of microalgae. This work is an application of three generations of PDMS chips already manufactured in IMS of Bordeaux1. In particular, the protocol is optimized from that previously proposed for E. coli bacteria [2], by using the real-time oscillating frequency due to mass loading during layers deposition : the microalgae is fixed onto the sensor surface coated with a polyelectrolyte multilayer (PEM), and the bioreceptor immobilization response time is greatly reduced using the microfluidic set up.
\end{abstract}

Key words: acoustic sensor, AFM, heavy metals, PDMS Chips, Spirulina

\section{1- Introduction}

Some microalgae species can accumulate high concentrations of heavy metals in contaminated ecosystems [3]. That's why a dried biomass of Arthrospira platensis (commonly named Spirulina), a member of blue-green algae, has been chosen in this study as bioreceptor for a biosensor detecting metallic cations. Adsorption can be ascribed to electrostatic attractive interactions between the positive metallic ions and algal cell wall, containing carboxylic, amino, thio, hydroxo and hydroxy-carboxylic groups [4]. The presence of these macromolecules in Spirulina wall has been confirmed by FTIR [5].

In this study, the Spirulina immobilization on the sensor surface was monitored by the real time response with acoustic wave sensors in liquid medium.
Immobilization techniques applied to algae are well-known and date from the end of the eighties [6]. In 1996, Cassidy et al. [7] have already proposed a review of the environmental applications of immobilized cells (not only algae). Immobilization algae techniques can be primarily divided into two groups: "passive" and "active" immobilization [6]. In this study, immobilization of the Spirulina cells was performed on polyelectrolyte multilayers (PEM) coated on a surface using a layer by layer (LBL) method. The LBL assembly technique consists in the alternate deposition of polyanions and polycations from aqueous solutions to build ultrathin multilayered films on flat substrates [8]. In this work, three generations of milli- to microfluidic polymeric analysis chambers combined with highly sensitive acoustic Love wave delay-lines were employed and previously studied by Tarbague $\mathrm{H}$. et al [9]. In this innovative approach, Atomic Force Microscope 
(AFM) studies were performed on different areas of the sensor surface, to characterize the Spirulina cell attachment to the substrate of polyelectrolytes.

AFM is a powerful imaging tool that mechanically probes a surface with unprecedented resolution. It allows investigation of conductive and non-conductive samples in various environments (such as in ambient conditions, vacuum or liquid). It is recognized as a valuable tool for studying biological materials and can be used to image cells under physiological conditions in a nondestructive manner. This tool can generate 3-D images of surfaces (topographic imaging) and provides information about surface properties such as adhesion properties and chemical composition (phase imaging) [10].

The AFM is based on the detection of small forces existing between a tip and an object. The tip is attached on one end of a cantilever which is "built in" at the other end, and the object is scanned along the tip with a piezo scanner. In this study, AFM images of Spirulina give informations of immobilized cells (shape, size, biovolume).

\section{2- Materials and Methods}

\section{2-1- Love Wave Sensor}

The Love wave sensor is an electromechanical sensor based on a guided shear horizontal surface acoustic wave (SH-SAW) mounted in oscillating configuration (frequency near $118 \mathrm{MHz}$ ), as previously described [9]. The sensor is made of two delay lines, each with metallic Interdigital Tranducers (IDT) deposited on a quartz substrate to generate and receive $\mathrm{SH}$ waves perpendicular to $\mathrm{X}$ crystallographic axis. A silicate guiding layer deposited on the entire structure allows confinement of the wave energy near the surface to maximize the sensor sensitivity.

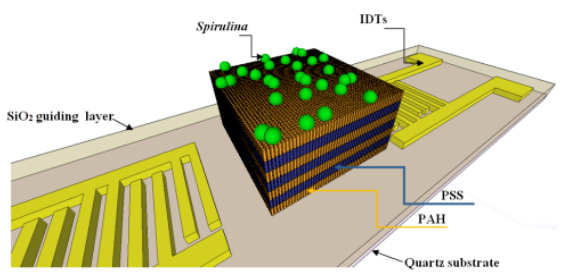

Fig.1. Scheme of a dual Love wave delay-line

The sensor surface is then coated with a polyelectrolyte (PE) multilayer of Poly (allylamine hydrochloride) (PAH cation) and poly (styrene sulfonate) (PSS anion). Finally, the microalgae (from a solution in TBS at $0.3 \mathrm{~g} / \mathrm{l}$ filtred by a $0.8 \mu \mathrm{m}$ filter) are immobilized by electrostatic adsorption of oppositely charged polymer chains (Fig.1), with an optimum flow rate at $80 \mu \mathrm{l} / \mathrm{min}$ and $40 \mu \mathrm{l} / \mathrm{min}$ with HDSU1 and HDSU2 respectively (see 2-2). The oscillating frequency of the acoustic sensor allowed realtime monitoring of the mass loading during layers deposition.

\section{2-2- PDMS Chips}

The analysis chamber, confining liquid on the sensitive sensor surface, is realized with Polydimethylsiloxane (PDMS) chips, and pressed on the sensor before functionalization [9]. Three generations were tested previously, the first one, HSSU used for Hydrostatic SetUp, consisted in getting the fluid sample with a micropipette in, then out of the open analysis chamber, resulting in diffusive approach of species towards the sensing surface (Fig.2) [9].

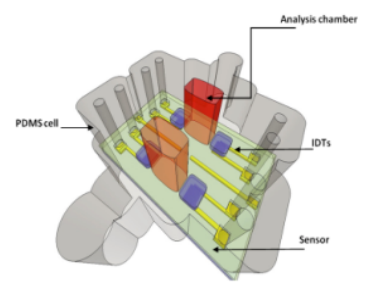

Fig.2. HSSU [9]

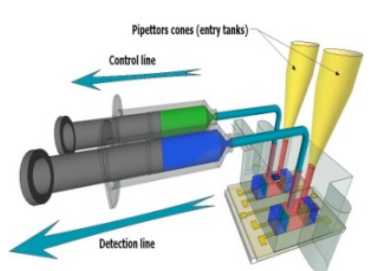

Fig.3. : HDSU1 [9]
The second and third chips were used for Hydrodynamic Set-Up (HDSU) consisting in flowing the fluid samples with a programmable syringe pump (BS8000, Bioseb) through the analysis chambers. They correspond to millifluidic [9] and microfluidic setups [2], and are called HDSU1 (Fig.3) and HDSU2 (Fig.4) respectively. For the HDSU1 setup, the analysis chamber is closed with integrated holes to inject liquid. For the HDSU2 one, the analysis chamber is replaced by a microfluidic chamber with microfluidic networks, enhancing a homogeneous and controlled flow on the sensitive path of the propagating acoustic wave.

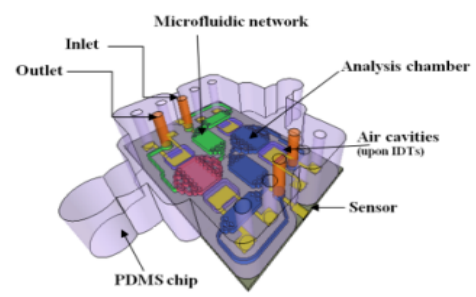

Fig.4. HDSU2 [9]

\section{2-3- AFM device}

AFM experiments were performed on the NSI platform of LOMA (Bordeaux1) using a Bioscope II mounted on an Olympus inverted optical microscope and operating with the NanosCope V controller (Veeco-Brucker, Santa 
Barbara, CA). This AFM is equipped with a $G$ scanner (maximum $X Y$ scan range of $150 \mu \mathrm{m} * 150 \mu \mathrm{m}$ with vertical range $Z$ of $12 \mu \mathrm{m}$ ). AFM experiments were achieved in air and in liquid with contact and tapping mode. For each experiment, three images are recorded at the same time: trace and retrace height images, deflection images (signal error) and friction images in contact and phase images in tapping. Only the trace height image is shown in this paper. For more clarity, images are flattened. Force Curves (FC) were obtained with the same cantilever in the force calibration mode and collected by measuring the cantilever deflection as the sample was moved toward the tip.

\section{3- Experimental Results}

The different set-ups were used with the same concentration of Spirulina in TBS solution. A frequency decrease was recorded after each $P E$ coating step and signal stabilization was achieved before the Spirulina immobilization. A typical real-time response is shown on Fig.5(a).

A frequency decrease was real-time recorded during microalgae immobilization with HDSU setup (Fig.5(b)), whereas no response could be observed with the HSSU one within a few hours (Fig.5(a)). This difference was attributed to an improved interaction rate between the PELcoated surface and Spirulina, due to the fluid flow which promotes hydrodynamical, rather than only diffusive, transport effects.

With HDSU2 setup compared to HDSU1 one, the frequency shift reaches a greater steadystate value, with reduced response time $\zeta_{90 \%}$. As shown in Fig.6, it decreased from $80 \mathrm{~min}$ with HDSU1 to 30 min with HDSU2. This is due to the advective transport coupled to a better fluid confinment [9].

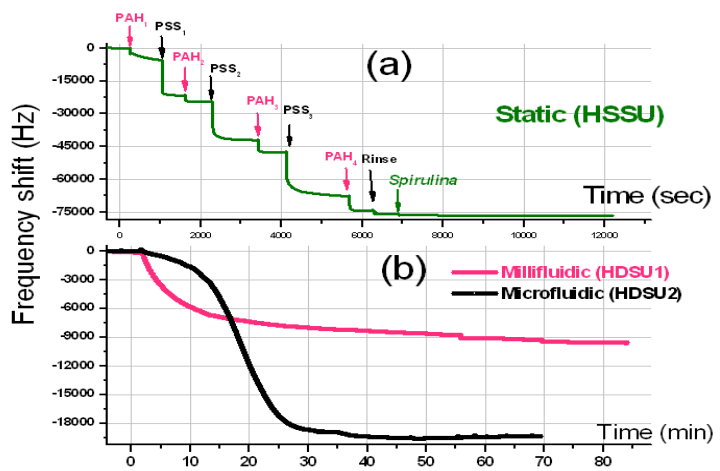

Fig.5. Typical real-time responses of the acoustic sensor (a) with HSSU during PEM and Spirulina immobilization, (b) real time frequency with HDSU during Spirulina immobilization.

A reproducible delay at the origin of the curve with HDSU2 setup (Fig.5(b)) may be attributed to mechanical forces induced by the flow on the PEM-coated surface and on microalgae near the surface. Further experiments are undergoing to better understand, possibly suppress, this phenomenon, whereas it did not affect efficiency of the resulting sensor for heavy metal detection.

In conclusion, the microfluidic configuration (HDSU2) reduced significantly the time required for the Spirulina immobilization by favoring their movement homogeneously close to the entire surface, thus improving their immobilization.

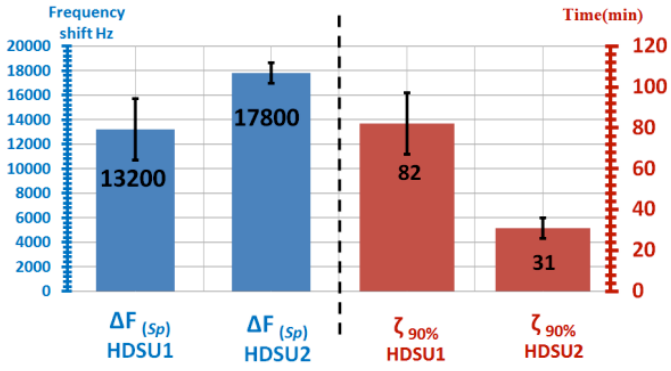

Fig.6. Steady-state frequency shift and response time of sensor responses to Spirulina immobilization, with both hydrodynamic HDSU1 and HDSU2; mean values and error bars have been calculated from 5 experiments with different delay-lines.

This remarkable evolution in kinetics detection is an important factor to improve the detection limit during the injection of heavy metals in solution. Thus, this microfluidic system has been used to transport metal cations towards the biorecepting film of cells of Spirulina. An experimental detection limit inferior to $10^{-12} \mathrm{M}$ has been obtained with heavy metals, and lysis of Spirulina above $10^{-3} \mathrm{M}$ has been observed [11].

\section{4- AFM imaging}

The topographic study revealed homogeneous layers of polyelectrolytes, even at larger scales (Fig.7a-b). Spirulina cells have been immobilized using HDSU2.
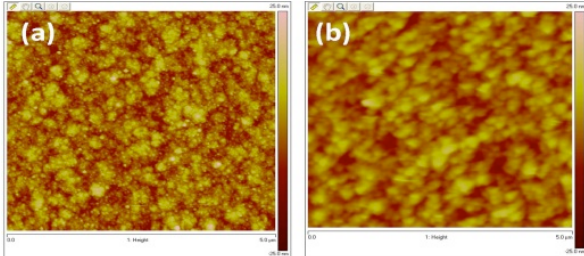

Fig.7. 2D Height AFM images obtained in tapping mode in air (a) SAW sensor (b) PEM substrate.

Several populations were observed: single cells whose average size was about $0.8 \mu \mathrm{m}$ (Fig.8a) while other cells were clustered in groups of two or three (Fig.8b). Study by varying different parameters shows that cells were firmly attached to the microsensor surface. The force 
curves results obtained at the very top of the cells and from which one can deduce the elastic modulus were in good agreement with these observations (Fig.9).
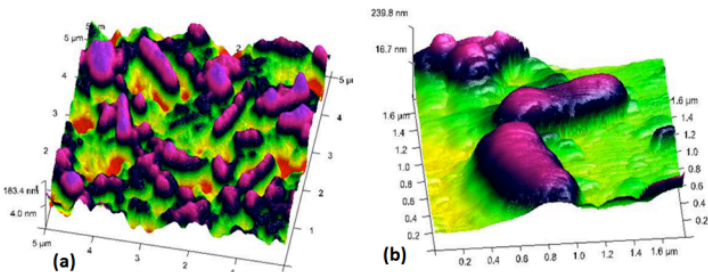

Fig.8. Three dimensional AFM height images obtained in contact mode and in liquid $(0,15 M$ TBS solution) showing immobilized Spirulina cells

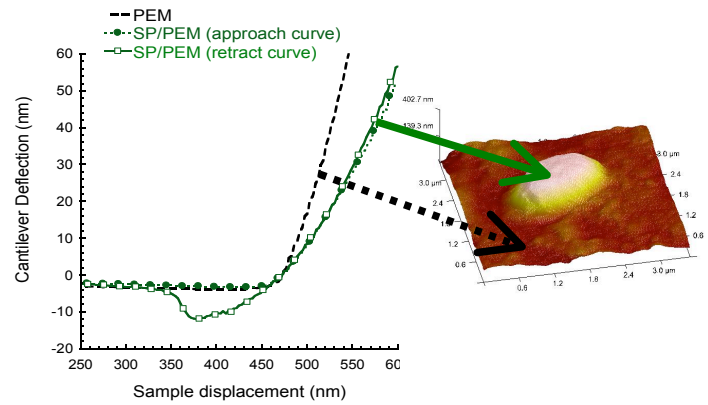

Fig.9. Typical experimental force curves (FC) obtained with the same cantilever on the studied samples in liquid. The slope of the force curve containing the mechanical properties of the surface studied is compared indicating an elastic cell behavior.

\section{Conclusion}

An optimization of Spirulina biofilm based Love wave microsensor was presented using biocompatible PDMS chip calibrated for reproducibility of experimental conditions. Microfluidic network improves the efficiency of interactions between PEM and Spirulina with high speed immobilization. In the curve force obtained on microalgae cell, the linear portion of the curve represents a linear elastic deformation of the Spirulina surface. This shows that cells are firmly attached to the microsensor surface.

\section{Acknowledgements}

This work was financially supported by the CMCU, project No.10G1103. PDMS chips were designed in the frame of ANR project BIOALERT. The authors also want to thank M. Benoît from LAAS-CNRS (Toulouse France) for processing of piezoelectric delay-lines within a collaborative project through the national technical realization network RTB.

\section{References}

[1] L. Fang, C. Zhou, P. Cai, W. Chen, X. Rong, K. Dai, W Liang, J. Gu and Q. Huang, Binding characteristics of copper and cadmium by cyanobacterium Spirulina platensis, Journal of Hazardous Materials., 190 (1-3),810-5 (2011); doi:10.1016/j.2011.10.038

[2] I. Gammoudi, H. Tarbague, J.-L. Lachaud, S. Destor, A. Othmane, D. Moynet, R. Kalfat, D. Rebière, C. Dejous, Love wave bacterial biosensors and microfluidic network for detection of heavy metal toxicity, Sensor Letters, 9 (2), 816-819 (2011); doi: 10.1166/sl.2011.1621

[3] Z.-Y. Li, S.-Y. Guo, L. Li, Study on the process, thermodynamical isotherm and mechanism of $\mathrm{Cr}$ (III) uptake by Spirulina platensis, J. Food Eng. (75), 129-136 10.1016/j.jfoodeng.2005.04.003

(2006); doi:

[4] H.B. Xue ,W. Stumm, L. Sigg, The binding of heavy metals to algal surfaces, Water Res. (22), 917-926 (1988)

[5] N. Tekaya, N. Sakly, Haf. Ben Ouada, Hat. Ben Ouada, N. Jaffrezic-Renault, and F; Lagarde, Impedimetric Characterization of Alginate Entrapped Arthrospira Platensis at a Platinum/Electrolyte Interface. Effect of Cadmium Ions, Sensor Letters (9), 1-5 (2011);

[6] M.-G. Ignacio, Microalgae immobilization: Current techniques and uses. Bioresource Technology (99) 3949-3964 (2008); doi: 10.1016/j.biortech.2007.05.040

[7] M.B. Cassidy, H. Lee, J.T. Trevors, Environmental applications of immobilized cells: a review. J. Ind. Microbiol. (16) 79-101 (1996); doi: 10.1007/BF01570068

[8] M. Schonhoff, Self-assembled polyelectrolyte multilayers, Current Opinion in Colloid and Interface Science (8) 86-95 (2003); doi: 10.1016/S1359-0294(03)00003-7

[9] H. Tarbague, J.-L. Lachaud, S. Destor, L. Vellutini, J.-P. Pillot, B. Bennetau, E. Pascal, D. Moynet, D. Mossalayi, D. Rebière, C. Dejous, PDMS (Polydimethylsiloxane) microfluidic chip molding for love wave biosensor, Journal of Integrated Circuits and Systems V5. N2. (2010); doi: $10.1149 / 1.3183735$

[10] A. Simon, T. Cohen-Bouhacina, M. C. Porte, J. P. Aime, J. Amédée, R. Bareille and C. Baquey, Characterization of Dynamic Cellular Adhesion of Osteoblasts Using Atomic Force Microscopy" Cytometry Part A 54A:36-47 (2003); doi: 10.1002/cyto.a.10052

[11] N. Tekaya, I. Gammoudi, H. Tarbague, F. Moroté, V. Raimbault, N. Sakly, Hat. Ben Ouada, Haf.Ben Ouada, F. Lagarde, D. Rebière, N. Jaffrezic-Renault, T. Cohen-Bouhacina, C. Dejous, accepted in Biosensors 2012, May 1518, 2012, Cancun, Mexico. 